\title{
Rasgos de personalidad, edad, cultura y restricciones hídricas y dietéticas en pacientes en hemodiálisis
}

\author{
Ana Maria Ruiz Vaca* - Victoria Eugenia Muñoz García* - Jose Luis García Vega** - Nayet Mohamed \\ Maanan* - Juana García Criado* - Aziza Bagdad Abselam*
}

\section{*DUE Unidad de Diálisis del Hospital Comarcal de Melilla \\ **DUE-PSICOLOGO CLÍNICO; Delegación de Defensa de Melilla}

\section{Resumen}

Queda mucho por investigar y comprender alrededor de la interacción entre lo físico y lo mental. Los pacientes sometidos a hemodiálisis debido a su enfermedad, deben cumplir una serie de normas dietéticas, de cuidado de su acceso vascular, etc. Pensamos que los rasgos de la personalidad, la edad, la cultura, pueden influir en la aceptación de la enfermedad de nuestros pacientes renales en tratamiento sustitutivo con hemodiálisis, y por lo consiguiente en el cumplimiento de las normas dietéticas, lo cual se vería reflejado en sus resultados analíticos pre-HD (niveles de potasio, de fósforo, ganancia interdiálisis), aunque también somos conscientes de que pueden influir otras variables, como es el nivel de afectación renal, nivel sociocultural, etc.

La muestra del estudio la constituyeron 35 pacientes de la unidad de diálisis de Melilla, de diferentes edades (mayores de 18 años) y de ambos sexos. Para conocer los rasgos de personalidad de nuestros pacientes, se les realizó un cuestionario validado de personalidad.

Los datos registrados fueron: edad, sexo, cultura, rasgo de personalidad, potasio, fósforo, ganancia interdiálisis.

Correspondencia:

Ana María Ruiz Vaca

C/Mallorca, portal 1,30 A.Edificio Mizar 1

52006 Melilla

E-mail:amvr2368@hotmail.com
No hemos hallado correlación significativa entre los rasgos de personalidad, la cultura frente a la ganancia de peso interdiálisis, niveles de fósforo y potasio, pero sí hemos hallado correlación significativa negativa entre la variable edad frente a la ganancia interdiálisis y los niveles de fósforo, aunque no así en los niveles de potasio, dé tal manera que se ve reflejado que a mayor edad, presentan menos ganancia interdiálisis y menos niveles de fósforo.

\section{PALABRAS CLAVE:}

- HEMODIÁLISIS

- RASGOS DE PERSONALIDAD

- FÓSFORO

- POTASIO

- GANANCIA INTERDIÁLISIS

Personality traits, age, culture and hydric and dietetic restrictions in patients undergoing haemodialysis

\section{Abstract}

Much has still to be investigated and understood about the interaction between the physical and the mental. Patients undergoing haemodialysis because of their illness have to comply with a series of dietetic rules, care of their vascular access, etc. We think that personal traits, age, culture can influence the acceptance of the illness by our renal patients 
receiving substitute treatment with haemodialysis, and therefore the compliance with dietetic rules, which would be reflected in their pre-HD analysis results (levels of potassium, phosphorous, interdialysis weight gain), although we are also aware that other variables can have an influence, such as the level of renal affectation, sociocultural level, etc.

The sample in the study was made up of 35 patients of the dialysis unit in Melilla, of different ages (over 18) and of both sexes. To determine the personality traits of our patients, a validated personality questionnaire was used.

The data recorded were: age, sex, culture, personality trait, potassium, phosphorous, interdialysis weight gain.

We have not found a significant correlation between personality traits, culture compared to interdialysis weight gain, phosphorous and potassium levels, but we did find a significant negative correlation between the age variable and interdialysis weight gain and phosphorous levels, although not in potassium levels, so that the older the patient, the lower the interdialysis weight gain and the phosphorous levels.

\section{KEY WORDS: \\ - HAEMODIALYSIS \\ - PERSONALITY TRAITS \\ - PHOSPHOROUS \\ - POTASSIUM \\ - INTERDIALYSIS WEIGHT GAIN}

\section{Introducción}

Hasta hace muy pocos años la medicina tradicional defendía una separación radical entre cuerpo y mente, hoy sabemos que el individuo es un conjunto único y global. Las personas reaccionamos ante situaciones objetivamente idénticas, de forma completamente distinta, diferencias individuales que se explican desde la variable de la personalidad ${ }^{1}$ y su interacción con el medio ambiente ${ }^{2}$, constituyendo en conjunto un modo único de sentir, pensar y actuar. En nuestra unidad de diálisis vemos diariamente como hay pacientes que vienen sesión tras sesión con gran exceso de peso y como en la analítica mensual hay pacientes que presentan valores altos de fósforo y potasio a pesar de estar bien informados sobre el tipo de dieta a seguir así como de la cantidad de líquido que pueden ingerir (diuresis total en $24 \mathrm{~h}+500 \mathrm{cc}$ ), mientras que hay otros pacientes que sin embargo traen poco peso y sus valores analíticos del fósforo y potasio, están más controlados, es decir presentan una buena adherencia al tratamiento , tanto al tratamiento dietético como al farmacológico (quelantes del fósforo).

Por adherencia al tratamiento desde el modelo biomédico, entendemos:

Al cumplimiento o la obediencia del paciente para seguir las instrucciones y prescripciones del profesional de la salud para conseguir el resultado preventivo 0 terapéutico deseado (Meichenbaum y Turk, 1987)³.

La adherencia terapéutica según la OMS (2004) es influida simultáneamente por varios factores:

- Factores sociales y económicos

- El equipo o sistema de asistencia sanitaria

- Las características de la enfermedad

- Los tratamientos

- Los factores relacionados con los pacientes, como son:

- Los recursos

- Los conocimientos

- Las actitudes y las creencias

- Las percepciones y las expectativas del pacien$\mathrm{te}^{4}$.

La personalidad fue definida por Eysenk como la suma total de patrones conductuales y potenciales del organismo, determinados por la herencia y por el medio social ${ }^{5}$. Los rasgos de personalidad según el modelo europeo es tridimensional, por lo que nos encontramos con: la introversión-extraversión, neuroticismo (emotividad) y el psicoticismo (dureza). Los rasgos de la personalidad, no se observan directamente, sino que lo que se observa es la conducta manifiesta6.

Partiendo del modelo de personalidad desarrollado por Eysenk y Eysenk (1964), en el que se analizaron las conductas que caracterizan a los extravertidos y a los 
introvertidos, definieron al extravertido típico como un ser sociable, al que le gustan las fiestas, tiene muchos amigos, se arriesga, es despreocupado, le gustan las bromas y su lema es diviértete y sé feliz, mientras que el introvertido típico es un individuo tranquilo, retraído, reservado, distante con los demás excepto con los amigos íntimos, cauteloso, con elevado control emocional, suele ser previsor, "mira antes de saltar, disfruta de un modo de vida ordenado.

Las personas que tienden a la inestabilidad emocional, suelen ser ansiosos, preocupados, presentan reacciones muy fuertes a todo tipo de estímulos y le cuesta volver a la normalidad después de cada experiencia que provoca una elevación emocional. Sus fuertes reacciones emocionales le interfieren para lograr una adaptación adecuada mientras que las personas que son más estables emocionalmente hablando, vuelven a su estado habitual rápidamente después de una elevación emocional, normalmente es equilibrado, calmoso, controlado ${ }^{7}$.

Las enfermedades crónicas como la enfermedad renal sometida a tratamiento sustitutivo, provocan fuertes reajustes en la vida del individuo ${ }^{8}$, lo que conlleva unas pautas de comportamiento que se van a adaptar de una u otra forma dependiendo de los rasgos de personalidad, edad, etc. Estos reajustes van desde acudir a las sesiones, la puntualidad en las mismas, el cuidado de la fístula, así como el control hídrico y dietético ${ }^{8}$.

El cumplimiento de las normas de control hídrico y dietético, por parte de los pacientes renales, repercute en una menor morbilidad e incremento de la esperanza de vida de estos pacientes 9 .

En este trabajo hemos querido focalizar especialmente la posible importancia de los rasgos de la person alidad así como la edad, la cultura en relación con el cumplimiento de las normas de restricción hídrica y dietética de la I.R.C. sometida a hemodiálisis.

\section{Objetivo}

Valorar si existe influencia de los rasgos de personalidad, edad y cultura, sobre los resultados analíticos pre-hemodiálisis (potasio, fósforo) así como la ganancia interdiálisis relacionados con el cumplimiento del control hídrico y dietético en los pacientes de nuestra unidad.

\section{Material y método}

Llevamos a cabo un estudio descriptivo, transversal y de correlación teniendo en cuenta la ganancia interdiálisis media y los valores de fósforo y potasio que presentaron los pacientes en el mes de enero de 2010, así como la edad y la cultura.

Tras solicitar y obtener permiso de forma verbal a 35 pacientes $(56,5 \%)$ de nuestra unidad, excluyendo a una paciente menor de 15 años (1,6\%), por no cumplir los requisitos de edad, otros tres pacientes por no encontrarse en ese periodo en la ciudad $(4,8 \%)$, y el resto de pacientes por motivos de barrera idiomática (37\%). Se realiza un cuestionario para conocer los rasgos de personalidad de nuestros pacientes sometidos a hemodiálisis. El cuestionario utilizado es el EPQ-A de EYSENCK en su adaptación española, realizada por Estudios de TEA Ediciones que valora tres dimensiones básicas de la personalidad (extraversión, neuroticismo y psicoticismo, así como una escala de sinceridad). Es uno de los cuestionarios más utilizados para evaluar la personalidad. Los rasgos de personalidad están sujetos a una base genética y se consideran un continuo en las personas, las cuales se pueden acercar 0 alejar de los extremos en un mayor 0 menor grado $^{10}$. Consta de 96 preguntas con respuestas dicotómicas de sí o no. Los resultados fueron corregidos por un psicólogo que utilizó las plantillas específicas para ese cuestionario según la edad y sexo.

Se realizó la recogida de las siguientes variables: edad, sexo, cultura, rasgo de personalidad, potasio, fósforo, ganancia interdiálisis. Los valores de fósforo y potasio fueron recogidos de la analítica que mensualmente se les realiza a los pacientes (específicamente fue en el mes de enero de 2010), mientras que los valores de ganancia interdiálisis se recogieron haciendo la media de la ganancia interdiálisis durante ese mes, que aparecían en los informes médicos de cada paciente.

Se definió como mala adherencia a la restricción de líquidos una ganancia de peso interdialítico $\geq 1,5 \mathrm{~kg}$, aunque hemos visto que en otros artículos también utilizan como criterio una ganancia $>1 \mathrm{~kg} 0>2,5 \mathrm{~kg}$. En cuanto a la valoración de la restricción dietética, se utilizó como corte un nivel de potasio sérico prediálisis $\geq 5,5 \mathrm{mmol} / \mathrm{L}$, siendo éste el valor más usado en las bibliografías consultadas ${ }^{11} \mathrm{y}$ un nivel de fósforo de $\geq 5,5 \mathrm{mg} / \mathrm{dl}$ ya que en la guía KDOQI aconsejan que los valores de fósforo en los pacientes renales sometidos a diálisis debe de estar 
comprendido entre 3,5 y 5,5 mg/dl para prevenir el hiperparatiroidismo secundario. Posteriormente se creó una base de datos y se analizaron los resultados mediante el programa estadístico SPSS 15 . Se realizó la prueba de Kolmogorov para comprobar que las variables seguían a la distribución normal. Se realizó la prueba t de Student de una muestra para la comparación de medias, así como también se analizó la correlación existente entre las distintas variables. Se han considerado estadísticamente significativas las $p$ inferiores a 0,05.

\section{Resultados}

Analizando los resultados de los 35 pacientes con una edad media de 55,2 años, siendo el rango entre 24 y 82 años, realizamos cuatro tablas para valorar los resultados y observamos:

En la tabla 1 podemos apreciar la frecuencia de las distintas variables agrupadas por el sexo, en ella nos llama la atención que el número de pacientes inestables es mucho mayor que el de estables (gráfico 1), quizás precipitada esta inestabilidad emocional por el stress provocado por la enfermedad renal crónica, que puede actuar como factor desencadenante.

\begin{tabular}{|c|l|l|}
\hline \multicolumn{1}{|c|}{ variables } & \multicolumn{1}{|c|}{ N } & \multicolumn{1}{c|}{$\%$} \\
\hline $\begin{array}{c}\text { Sexo } \\
\text { Hombres }\end{array}$ & 35 & 51,4 \\
\hline Mujeres & 18 & 48,6 \\
\hline $\begin{array}{c}\text { Cultura } \\
\text { Cristiana }\end{array}$ & 17 & \\
\hline Musulmana & 13 & 37,1 \\
\hline $\begin{array}{c}\text { R. personalidad } \\
\text { Extravertido }\end{array}$ & 22 & 62,9 \\
\hline hombres & 21 & \\
\hline mujeres & 12 & 60 \\
\hline Introvertido & 9 & 66,7 \\
\hline hombres & 14 & 52,9 \\
\hline mujeres & 6 & 40 \\
\hline Estables & 8 & 33,3 \\
\hline hombres & 11 & 47,1 \\
\hline mujeres & 5 & 31,4 \\
\hline Inestables & 6 & 27,8 \\
\hline Hombres & 24 & 35,3 \\
\hline mujeres & 13 & 68,6 \\
\hline & 11 & 72,2 \\
\hline
\end{tabular}

Tabla 1: Tabla de frecuencia de las distintas variables agrupadas por el sexo

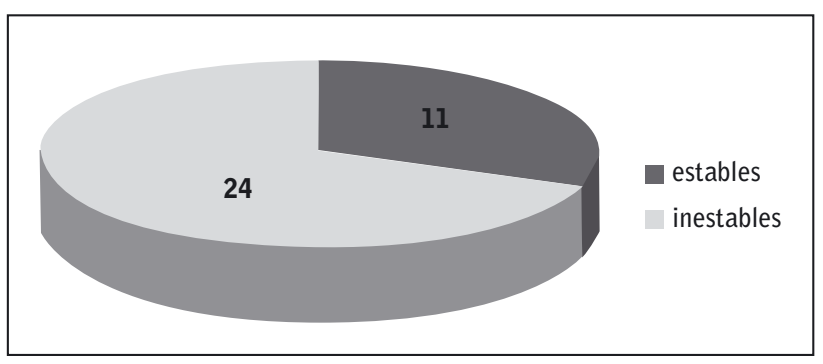

Gráfico 1: Porcentaje de pacientes estable versus inestables.

En la tabla 2, se enumeran las distintas medias y desviaciones típicas de las variables que utilizamos para medir la adherencia dietética e hídrica, así como de la edad de la muestra, no siendo los datos significativos, ya que son muy similares los resultados, pero aun así, se percibe que los extravertidos, inestables y musulmanes, tienen una ganancia interdiálisis media superior al resto.

En la tabla 3 se representan los coeficientes de correlación de Pearson entre los indicadores de adhesión al tratamiento hídrico y dietético con la edad, siendo significativo y negativo poniendo de manifiesto que la edad influye en la ganancia de peso interdiálisis y los niveles de fósforo de nuestros pacientes, por lo consiguiente rebela que los pacientes de mayor edad son más cumplidores que los más jóvenes.

En la tabla 4, constatamos que el $66,7 \%$ de los pacientes extrovertidos traían más de 1500 gr por sesión, frente al 50\% de los pacientes introvertidos. El 54,5\% de los pacientes estables, traían más de $1500 \mathrm{gr}$ de peso interdiálisis frente al $62,5 \%$ de los paciente inestables. No hemos encontrado correlación significativa, aunque se puede apreciar que es mayor el tanto por ciento de pacientes extravertidos e inestables que traen más peso (gráfico 2 ). El 23,8\% de los pacientes extravertidos y el $7,1 \%$ de los pacientes introvertidos, tenían más de $5,5 \mathrm{mmol} / \mathrm{l}$ de potasio .El $20,8 \%$ de los pacientes inestables y el $9,1 \%$ de los pacientes estables tenían valores superiores a 5,5 mmol/I.El 38,1\% de los pacientes extravertidos y el $35,7 \%$ de los pacientes introvertidos presentaban valores de fósforo superior a $5,5 \mathrm{mg} / \mathrm{dl}$.El $41,7 \%$ de los pacientes inestables y el $27,3 \%$ de los pacientes estables tenían valores de fósforo superior a 5,5 mg/dl. 
[ A.M. Ruiz Vaca, et al ]

Rasgos de personalidad, edad, cultura y restricciones hídricas y dietéticas en pacientes en hemodiálisis

\begin{tabular}{|c|c|c|}
\hline Variable & Media & Desviación típica \\
\hline \multicolumn{3}{|c|}{ Ganancia interdiálisis } \\
\hline Hombre & 1936,1 & 1062,7 \\
\hline Mujer & 1785,2 & 824,9 \\
\hline Extravertido & 1928,6 & 1015,9 \\
\hline Introvertido & 1764,2 & 851,1 \\
\hline Estable & 1668,2 & 1081 \\
\hline Inestable & 1952,1 & 884,4 \\
\hline Cristiana & 1729,2 & 989,4 \\
\hline Musulmana & 1947,7 & 928,7 \\
\hline Edad(años) & 55,2 & 15,5 \\
\hline Hombre & 56,2 & 15,5 \\
\hline Mujer & 54,2 & 15,9 \\
\hline Extravertido & 55,1 & 14,7 \\
\hline Introvertido & 55,3 & 17,2 \\
\hline Estable & 54,9 & 14,7 \\
\hline Inestable & 55,4 & 17,2 \\
\hline Cristiana & 60,1 & 14,3 \\
\hline Musulmana & 52,7 & 15,8 \\
\hline \multicolumn{3}{|l|}{ Fósforo(mg/dl) } \\
\hline Hombre & 4,9 & 1,2 \\
\hline Mujer & 4,9 & 1,3 \\
\hline Extravertido & 4,9 & 1,1 \\
\hline Introvertido & 4,7 & 1,4 \\
\hline Estable & 4,7 & 1,4 \\
\hline Inestable & 4,7 & 1,2 \\
\hline Cristiana & 5,1 & 1,2 \\
\hline Musulmana & 4,7 & 1,3 \\
\hline \multicolumn{3}{|l|}{ Potasio(mmol/l) } \\
\hline Hombre & 4,7 & 0,6 \\
\hline Mujer & 5,2 & 0,9 \\
\hline Extravertido & 4,9 & 0,9 \\
\hline Introvertido & 4,9 & 0,7 \\
\hline Estable & 5,0 & 0,9 \\
\hline Inestable & 4,9 & 0,8 \\
\hline Cristiana & 5,0 & 0,6 \\
\hline Musulmana & 4,9 & 0,9 \\
\hline
\end{tabular}

Tabla 2: Frecuencia de medias y desviaciones típicas de las distintas variables que utilizamos para medir la adherencia hídrica y dietética, así como la edad

\begin{tabular}{|l|l|l|}
\hline Variable & $\begin{array}{l}\text { Correlación de } \\
\text { Pearson }\end{array}$ & $\mathbf{P}$ \\
\hline Edad/ganancia-interdiálisis & $-0,365$ & $0,031^{*}$ \\
\hline Edad/potasio & $-0,143$ & 0,413 \\
\hline Edad/fósforo & $-0,438$ & $0,009^{* *}$ \\
\hline
\end{tabular}

* La correlación es significativa al nivel 0,05 (bilateral)

** La correlación es significativa al nivel 0,01 (bilateral)

Tabla 3: Representación de los coeficientes de correlación entre los indicadores de adhesión al tratamiento y la edad

\begin{tabular}{|c|c|c|}
\hline Variable & $\mathbf{N}$ & $\%$ \\
\hline \multicolumn{3}{|c|}{ ganancia >1500gr } \\
\hline Extravertidos & 14 & 66,7 \\
\hline Introvertidos & 7 & 50 \\
\hline Estables & 6 & 54,5 \\
\hline Inestables & 15 & 62,5 \\
\hline \multicolumn{3}{|c|}{ ganancia <1500gr } \\
\hline Extravertidos & 7 & 33,3 \\
\hline Introvertidos & 7 & 50 \\
\hline Estables & 5 & 45,5 \\
\hline Inestables & 9 & 37,5 \\
\hline \multicolumn{3}{|c|}{ Potasio $>5,5 \mathrm{mmol} / \mathrm{l}$} \\
\hline Extravertidos & 5 & 23,8 \\
\hline Introvertidos & 1 & 7,1 \\
\hline Estables & 1 & 9,1 \\
\hline Inestables & 5 & 20,8 \\
\hline \multicolumn{3}{|c|}{ Potasio $<5,5 \mathrm{mmol} / \mathrm{l}$} \\
\hline Extravertidos & 16 & 76,2 \\
\hline Introvertidos & 13 & 92,9 \\
\hline Estables & 10 & 90,9 \\
\hline Inestables & 19 & 79,2 \\
\hline \multicolumn{3}{|c|}{ Fósforo $>5,5 \mathrm{mg} / \mathrm{dl}$} \\
\hline Extravertidos & 8 & 38,1 \\
\hline Introvertidos & 5 & 35,7 \\
\hline Estables & 3 & 27,3 \\
\hline Inestables & 10 & 41,7 \\
\hline \multicolumn{3}{|c|}{ Fósforo $<5,5 \mathrm{mg} / \mathrm{dl}$} \\
\hline Extravertidos & 13 & 61,9 \\
\hline Introvertidos & 9 & 64,3 \\
\hline Estables & 8 & 72,7 \\
\hline Inestables & 14 & 58,3 \\
\hline
\end{tabular}

Tabla 4: Porcentaje de pacientes según los rasgos de la personalidad que son más cumplidores o menos cumplidores 


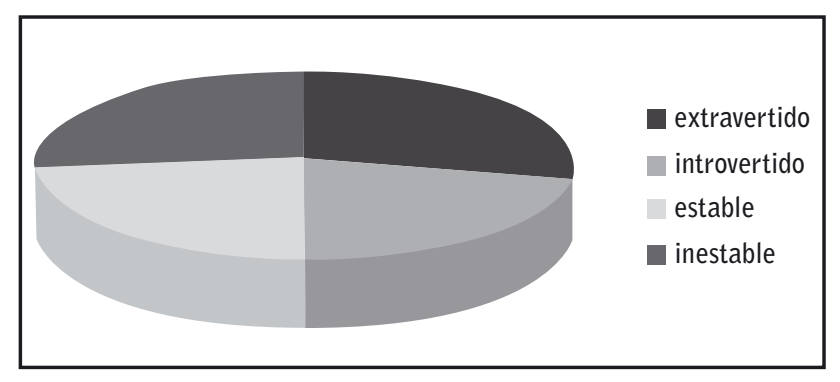

Gráfico 2: Representación del porcentaje de pacientes que tenían más de 1500 gr de ganancia interdiálisis de media.

\section{Discusión}

Como decíamos en nuestra introducción, hay que romper el dualismo mente / cuerpo, biología /cultura, ya que está más que demostrado que están íntimamente relacionados y las investigaciones científicas apuntan cada vez más a la necesaria y mutua relación entre nuestras formas de vivir (alimentación, etc.) y nuestro organismo y viceversa. Hay dos maneras en que la personalidad parece afectar la salud física: la primera se relaciona con el comportamiento y la segunda tiene que ver con la forma en que la persona percibe el mundo y reacciona ante él ${ }^{12}$. El comportamiento de los pacientes renales sometidos a hemodiálisis influye en su enfermedad, en la aparición de complicaciones, por eso éste debe ir encaminado a la aceptación de las normas, tanto dietéticas como farmacológicas que se le indica.

La bibliografía encontrada sobre el incumplimiento terapéutico, enfocado en detectar las causas por la que pacientes sometidos a hemodiálisis tienen una mala adherencia al tratamiento tanto farmacológico como de restricción de dieta y líquidos, cada vez es más abundante, en la que se visualiza que hay un alto porcentaje de pacientes que son incumplidores por no conocer bien el tratamiento farmacológic $0^{9}$ a pesar de recibirlo escrito por parte de los médicos todos los meses y recibir explicaciones añadidas de enfermería, o bien debido a la asociación de estados depresivos 9,13 que suelen afectar a estos pacientes.

Los rasgos de la personalidad de nuestros pacientes con su base genética y el factor ambiental, puede que influya en su comportamiento, en su adaptación a las restricciones que esta enfermedad impone y por lo tanto en sus valores pre-hemodiálisis de fósforo, potasio y la ganancia interdiálisis, aunque en nuestro estudio no ha quedado patente, quizás por ser una representación muestral pequeña, lo que deja abierta la posibilidad de futuras investigaciones en este campo. Pero sí se ha visto reflejado, al igual que en otros estudios sobre el mismo tema, que la edad es un factor concluyente a la hora de valorar el nivel de cumplimiento de los pacientes, siendo los pacientes de mayor edad los más cumplidores ${ }^{11}$.

\section{Conclusión}

Una de las mejores armas de la que disponen los pacientes para luchar contra las complicaciones de la IRC, es el tener una buena adherencia al tratamiento, tanto dietético como farmacológico, por lo tanto es muy importante el seguir trabajando en la educación sanitaria de nuestros pacientes para conseguir una adecuada concienciación sobre su enfermedad y sus repercusiones a corto y largo plazo.

Recibido: Diciembre 2010

Revisado: Diciembre 2010

Modificado: Abril 2011

Aceptado: Mayo 2011

\section{Bibliografía}

1. Personalidad y salud (acceso 5 mayo 2010).Disponible en:http://perso.wanadoo.es/aniorte_nic/apunt_ psicolog_salud_5.htm.

2. La personalidad, diferencias entre los individuos. (Acceso 5 mayo 2010). Disponible en:http://www. monografias.com/trabajos14/personalidad/personalidad.shtml.

3. Cepeda C, Wanner C, Barrales C, Núñez N. Locus de control y adherencia al tratamiento en personas con insuficiencia renal crónica. Ciencia Psicológica Vol. 1, N 1, 2007. Escuela de Psicología, Facultad de Ciencias Sociales, Universidad Central de Chile, 
ISSN 0718-4166(revista de internet). Disponible en: http://www.fcsucentral.cl/varios/files/file/publicaciones/cienciapsicologia_01/01_cienciapsicologica_01. pdf.

4. Ávila D. Adherencia a los tratamientos en enfermedades crónicas (0.M.S. 2004).Disponible en: http:// es.scribd.com/doc/6912000/ADHERENCIA-al-tratamiento

5.Personalidad-Multimania.Disponible en: http://ususrios.multimania.es/doliresa/index-2.hcml.

6. Bermúdez Moreno J. Psicología de la personalidad. Universidad de Educación a Distancia.Madrid. 1991.

7. Eysenk HJ, Eysenk SBG. Manual del cuestionario de personalidad para adultos. Madrid .TEA Ediciones; 1989.

8. Hemodiálisis. Fisterra salud .Disponible en: http:// www.fisterra.com/salud/3proceDT/hemodialisis.asp.

9. Martínez Aranda M A, Callejo Cano I, Arribas Cobo P, Fernández Fuentes A, Sáez Santolaya A J, García Estévez $S$, et al. Cumplimiento terapéutico de los pacientes de hemodiálisis. Libro de comunicaciones del XXXIV congreso nacional SEDEN. Hospal. Madrid 2009. Disponible en: www.seden.org/publicaciones. asp.
10. Marquina Parra D, Blasco Cabaña $C$, Mañé Buixo N, Ponz Clemente E, Martínez Ocaña JC, Yuste Jiménez E, García García M. Influencia de los rasgos de personalidad en la elección de la técnica de diálisis. Rev Soc Esp Enferm Nefrol. (Revista en Internet) 2005 enero-marzo (consultado 1 de mayo de 2010).Disponible en http://www.seden.org.

11. Iborra Moltó C, López-Roig S, Roca Alonso M, Pastor Mira MA. Adhesión al tratamiento y edad: variaciones en función de los parámetros utilizados. En Libro de comunicaciones del XXXIV congreso nacional SEDEN. Hospal. Madrid 2009. Disponible en: www.seden.org/publicaciones.asp.

12.Pesonalidad y enfermedad resistencia a las enfermedades. Disponible en:http://www.yerbasana.cl/?a=3773.

13. García Valderrama F W, Fajardo C, Guevara R, Gonzáles Pérez V y Hurtado A. Mala adherencia a la dieta en hemodiálisis: papel de los síntomas ansiosos y depresivos. NEFROLOGÍA. Vol. XXII. Número 3. 2002.Disponible en:http://www.revistanefrologia. com/revista/P1-E194/P1-E194-S132-A3499.pdf.

14. Moreno Sánchez V, del Amo Castaño R, Abad Casado Y, Andúgar Hernández J. Dieta y ejercicio físico en pacientes con insuficiencia renal crónica. Libro de comunicaciones del XXXII congreso nacional SEDEN. Hospal. Madrid 2008. Disponible en: www. seden.org/publicaciones.asp. 